\title{
Implementing community-based human papillomavirus self-sampling with SMS text follow-up for cervical cancer screening in rural, southwestern Uganda
}

\author{
Naima T Joseph ${ }^{1,2}$, Alexcer \\ Namuli $^{3}$, Bernard Kakuhikire ${ }^{4}$, \\ Charles Baguma ${ }^{4}$, Mercy \\ Juliet $^{4}$, Patience Ayebare ${ }^{4}$, \\ Phionah Ahereza ${ }^{4}$, Alexander \\ C Tsai ${ }^{2,4,5}$, Mark J Siedner ${ }^{2,5,6}$, \\ Thomas R Randall ${ }^{2,4,5,7}$, \\ Joseph Ngonzi ${ }^{3,4}$, \\ Adeline A Boatin ${ }^{2,4,5,7}$

\section{${ }^{1}$ Department of Obstetrics and Gynecology, Beth Isreal Deaconness Medical Center, Boston, MA, USA ${ }^{2}$ Harvard Medical School, Boston, MA, USA \\ ${ }^{3}$ Department of Obstetrics \& Gynecology, Mbarara Regional Referral Hospital, Mbarara, Uganda \\ ${ }^{4}$ Mbarara University of Science and Technology, Mbarara, Uganda \\ ${ }^{5}$ Center for Global Health, \\ Massachusetts General Hospital, Boston, MA, USA \\ ${ }^{6}$ Division of Infectious Disease, Department of Medicine, Massachusetts General Hospital, Boston, MA, USA \\ ${ }^{7}$ Department of Obstetrics \& Gynecology, Massachusetts General Hospital, Boston, MA, USA}

\section{Correspondence to:}

Adeline A. Boatin, MD, MPH Department of Obstetrics \& Gynecology Massachusetts General Hospital Harvard Medical School

Boston, MA, 02113

USA

Adeline_Boatin@mgh.harvard.edu
Background Self-collected HPV screening may improve cervical cancer screening coverage in low resource countries, yet data guiding implementation and follow-up of abnormal results are sparse.

Methods This is a prospective cohort implementation study of HPV self-testing program in Mbarara, Uganda with mobile phones to facilitate result notification and referral for treatment at a regional hospital. The effectiveness of the interventions was analyzed using Proctor's model of implementation. Women were interviewed following screening and at 6 months to assess acceptability and barriers to follow-up. Data were analyzed using descriptive statistics.

Results 159 of 194 (82\%) of eligible women underwent HPV self-sampling; of these, 27 (17\%) returned positive for high-risk HPV subtypes. We sent SMS messages providing test results and follow-up instructions to all participants. Seventeen (63\%) hrHPV-positive participants reported receiving SMS text instructions for follow-up, of whom 6 (35\%) presented for follow-up. The most common reasons for not returning were: lack of transportation $(n=11)$, disbelief of results $(n=5)$, lack of childcare $(n=4)$, and lack of symptoms $(n=3)$. Confidence in test results was higher for self-screening compared to VIA (Likert score 4.8 vs 4.4, $P=0.001$ ).

Conclusions Despite the use of SMS text-based referrals, only one-third of women presented for clinical follow-up after abnormal HPV testing.

Cervical cancer is a leading cause of preventable cancer morbidity and mortality, contributing approximately 250000 deaths in low- and middle-income countries (LMICs) annually [1]. The 90-79-90 campaign launched by the World Health Organization (WHO) aims to eliminate cervical cancer as a public health burden by vaccinating $90 \%$ of the eligible population, screening $70 \%$ of women and treating $90 \%$ of women in need by 2030 [2]. Despite the availability of effective screening methods for premalignant lesions (cytology, visual inspection with acetic acid (VIA), and human papillomavirus (HPV) testing), only 10\%-20\% of eligible women in LMICs are appropriately screened - largely due to barriers of patient access, technical expertise, laboratory capacity, and scalability [3-6]. Low-cost, easy to use, and scalable techniques for screening are needed to meet targets for the next decade.

Recognition of the strong causal relationship between persistent cervical infection with high-risk human papillomavirus (hrHPV) subtypes and cervical cancer has led to a proliferation of HPV molecular assays. Compared with VIA and cytology, hrHPV DNA testing has a higher sensitivity and negative predictive value for precancerous lesions, 
less inter-observer variability, a more favorable cost-effectiveness profile, and remains efficient in settings with high HPV vaccine penetration [7-10]. Importantly, hrHPV testing allows for self-collection of samples, which is accurate, feasible, and averts the logistical and human resource challenges associated with clinician-based screening [10-12]. For these reasons, hrHPV testing has been recommended by the WHO as a preferred screening strategy when feasible $[3,13]$.

Where cultural and program barriers limit women's access to clinician-based cervical cancer screening, homeor community-based self-sampling methods can increase participation among women who are difficult to reach through conventional means [14]. However, there are sparse guidelines on the implementation of hrHPV self-sampling in LMICs and limited data on the best methods to improve linkage to care and follow-up of HPV-positive women. Mobile phone text messages can be used to improve follow-up and participation in HIV in LMICs, but there are limited data on the role of text messaging to improve linkage to care after cervical cancer screening [15-17]. To address this knowledge gap, we leveraged a prospective cohort study with integrated community health fairs to pilot hrHPV self-testing in a community setting and implement mobile phone-based results notification to facilitate follow-up treatment at a regional cervical cancer prevention clinic.

\section{METHODS}

The parent study is a population-based sociocentric social network cohort in a rural administrative sub-unit of Rwampara District in southwestern Uganda, whose primary objective is to characterize the social network context of HIV stigma and understand its impacts on HIV care. Community feedback on study procedures is both formally and informally sought on a regular basis, through a community advisory board and multiple community sensitization meetings [18]. In response to community feedback, we incorporated cervical cancer screening (using VIA) into the study in 2017, leveraging the ongoing community health fair infrastructure to perform this pilot study of community-based hrHPV screening.

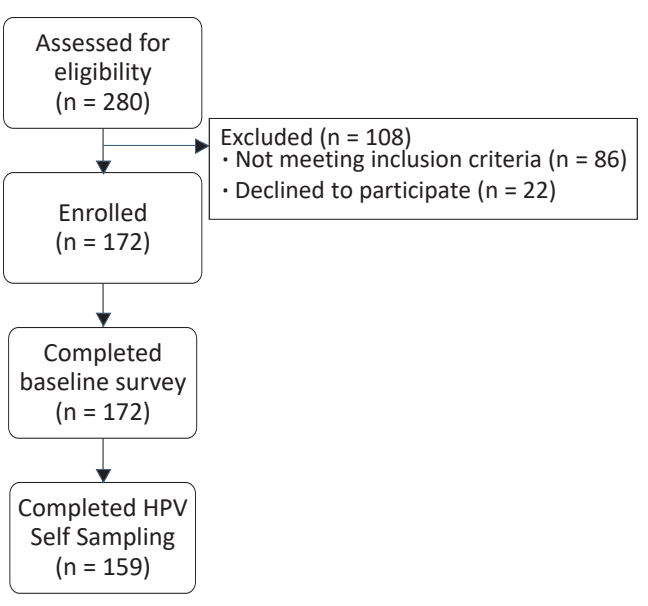

Figure 1. Flow diagram for patient recruitment, enrollment, and participation in the study.
Women aged 25- 65 years of age who were enrolled in the parent study were recruited to participate in the hrHPV pilot study. Attendees were recruited with a coordinated series of media and community outreach campaigns, including announcements delivered over the radio and from a mobile truck, banners displayed in public places, and leaflets distributed at church services. Community-wide sensitization meetings were held to provide information about the VIA screening and embedded hrHPV study and to answer questions. Eligible women were identified during these community meetings or at the time of the health fair and were referred to trained research assistants for a detailed explanation of the study and to obtain written informed consent. VIA was offered to eligible attendees independent of study participation. Women were excluded if they were (1) pregnant; (2) had a known gynecologic tract malignancy; or (3) had a prior hysterectomy (Figure 1).

During the health fair, all women received general education on cervical cancer prevention, which was adapted from the Alliance for Cervical Cancer Prevention "Planning and Implementing Cervical Cancer Prevention and Control" programmatic guide [13]. Structured interviews were used to gather information on past medical, reproductive, and sexual history, including prior cervical cancer preventive care. A literacy test was performed to assess study participants' ability to read short message service (SMS) text messaging. Cellular telephone contact information was obtained from each participant. Participants were then directed to the screening tents, where they received instructions for self-sampling and then collected the HPV sample. VIA screening was then performed by trained nurses and physicians, followed by cryotherapy for women meeting treatment criteria by VIA per WHO guidelines. Screen-positive women are eligible for cryotherapy if the entire lesion is visible, the squamocolumnar junction is visible, and the lesion does not cover more than $75 \%$ of the ectocervix [19].

Following screening with hrHPV self-sampling and VIA, participants were administered a post-screen survey to assess their experiences, including their overall impression, level of comfort, pain, embarrassment, and confidence associated with each screening test. Participants were provided contact information for the Cervical Cancer Prevention (CCP) clinic at Mbarara Regional Referral Hospital (MRRH). Women who screened positive or suspicious for cancer with VIA, or who had received cryotherapy, were instructed to present to the CCP for clinical follow-up. 
All samples were transported to a central laboratory at the MRRH. All HPV samples were then analyzed using GeneXpert Xpert ${ }^{\circledR}$ HPV (Cepheid, Sunnyvale, Calif.), which reports results as HPV 16, HPV 18/45, other hrHPV $(31,33,35,52,58 ; 51,59 ; 39,56,66,68)$, HPV negative, or invalid. Tests returning with invalid results were tested in triplicate.

Test results were delivered to women by SMS using an automated web-based algorithm developed by Innovation Streams Limited (Mbarara, Uganda) (Appendix S1 in the Online Supplementary Document). Pre-defined messages were scripted in Runyankore for women with HPV positive results and women with HPV negative results. Women who were HPV positive were instructed to follow-up at the CCP at MRRH. Women who were HPV negative were instructed to repeat cervical cancer screening in 5 years ( 1 year for women with HIV). Women who had a suspicious finding based on VIA were instructed to return at the health fair, and again through SMS instructions for follow-up, independent of HPV result, but were informed of their HPV test results.

Once test results were available, an automated SMS containing the scripted content was sent to the participant's phone number at no charge to the recipient. SMS text messages were sent monthly for a period of six months following the health fair. After six months, all participants received a follow-up phone survey, which we conducted in order to ascertain whether they had received and/or understood the SMS message, and/or to elicit their reasons for non-follow up (ie, for those participants who were either HPV positive or VIA positive and had not yet presented for care).

During the study period and following the health fair, nurses at the CCP kept a log of all returning participants. Participants who returned for follow-up underwent colposcopy, biopsy, and recommended treatment under the discretion of the clinic physician and nurses, and per WHO and Uganda cervical prevention guidelines [20]. Clinical chart abstraction to ascertain clinical care was performed for participants returning to clinic.

We aimed to understand the effectiveness of this intervention to achieve screening goals as well as to understand implementation outcomes for each component of the intervention. Thus we evaluated this pilot on both

Table 1. Summary characteristics of women who underwent HPV Self sampling $(\mathrm{n}=159)$

\begin{tabular}{lc} 
CHARACTERISTICS & N (\%) \\
Mean age in years (range) & $41.2(25-63)$ \\
\hline Literacy & $126(79)$ \\
\hline Relationship status: & $120(76)$ \\
\hline Married & $1(0.6)$ \\
\hline Partnered & $19(12)$ \\
\hline Widowed & $6(4)$ \\
\hline Single & $13(8)$ \\
\hline Divorced & $21.2(14-32)$ \\
\hline Mean age at first intercourse in years (range) & $111(71)$ \\
\hline Number of lifetime partners: & $34(22)$ \\
\hline $0-2$ & $11(7.0)$ \\
\hline $3-4$ & $1(0.6)$ \\
\hline $5-6$ & \\
\hline$\geq 7$ & \\
\hline Mean number previous pregnancies \pm SD (range) & $5.5 \pm 0.49(0-12)$ \\
\hline Mean number live births \pm SD (range) & $4.5 \pm 0.17(0-10)$ \\
\hline Postmenopausal & $46(29)$ \\
\hline Cervical cancer screening history: & $102(65)$ \\
\hline No prior screening & $37(66)$ \\
\hline Previous screening method: & $6(11)$ \\
\hline VIA & $9(16)$ \\
\hline Pap & $4(7)$ \\
\hline HPV & \\
\hline Unsure & \\
\hline Prior screen results: & \\
\hline Normal & \\
\hline Abnormal & \\
\hline Unsure & \\
\hline & \\
\hline
\end{tabular}

HPV - human papillomavirus, SD - standard deviation, VIA - visual inspection after acetic acid application implementation outcomes (penetration, appropriateness, acceptability and feasibility) and service outcomes (effectiveness) chosen from Proctor's model of implementation research [21]. Penetration was defined as the percentage of women meeting eligibility criteria for screening who consented to participate and had sample collection performed. Appropriateness was defined as the percentage of women with the literacy skills to read results via SMS and who had a phone to receive SMS results. Acceptability was measured using mean scores from the post-screen survey. Feasibility was defined as the percentage of women obtaining samples, having valid test results, and receiving SMS results. Effectiveness was defined as the number of women with positive results returning for follow-up in the CCP. Stata statistical software (version 16.1, College Station, TX, USA) was used for analyses.

This study was approved by the Institutional Review Boards at Mbarara University of Science and Technology, Mbarara Uganda, and Massachusetts General Hospital, Boston, MA. Study data were collected and managed using REDCap electronic data capture tools hosted at Partners Healthcare [22].

\section{RESULTS}

Over a 5-day period, 280 women were screened. Of these, 194 (69\%) met inclusion criteria, of whom 172/194 (89\%) were enrolled and completed the baseline survey and 159/194 (82\%) collected HPV self-samples, achieving penetrance of $79 \%$ of the intended population (Figure 1). Participating women had a mean age of 41.2 years (range, 25-63 years). Most women were married (120 [76\%]), had 2 or fewer lifetime partners (111 [71\%]), and had a mean age of first intercourse of 21.2 years (range, $14-$ 32 years) (Table 1 ). 
Table 2. SMS text interpretation and follow-up among HPV positive and HPV negative women (N, \%)

\begin{tabular}{|c|c|c|}
\hline OUTCOMES & $\begin{array}{l}\text { OVERALL } \\
(\mathrm{N}=159)\end{array}$ & $\begin{array}{c}\text { HPV } \\
\text { POSITIVE } \\
(\mathrm{N}=27)\end{array}$ \\
\hline \multicolumn{3}{|l|}{ VIA screening results: } \\
\hline Total screened with VIA & $138(87)$ & $27(100)$ \\
\hline VIA negative & $114(83)$ & $18(67)$ \\
\hline VIA positive & $14(10)$ & $4(15)$ \\
\hline Suspicious for cancer & $1(1)$ & 0 \\
\hline Unsatisfactory VIA & $8(6)$ & $1(4)$ \\
\hline Cryotherapy & $9(6)$ & $3(11)$ \\
\hline \multicolumn{3}{|l|}{ HPV test result: } \\
\hline Positive & $27(17)$ & - \\
\hline Negative & $129(81)$ & - \\
\hline Invalid & $4(2.5)$ & - \\
\hline \multicolumn{3}{|l|}{ HPV genotype: } \\
\hline 16 & - & $6(20)$ \\
\hline $18 / 45$ & - & $6(20)$ \\
\hline Other high risk & - & $21(78)$ \\
\hline Co-infection with one or more hrHPV strain & & $5(19)$ \\
\hline \multicolumn{3}{|l|}{ SMS text delivery: } \\
\hline \multicolumn{3}{|l|}{ Receipt of SMS text messages } \\
\hline Report receiving SMS text & $74(46.2)$ & $17(63)$ \\
\hline Report not receiving SMS text & $49(30.6)$ & $7(26)$ \\
\hline Unsure if received SMS text & $16(10.0)$ & $2(7)$ \\
\hline \multicolumn{3}{|l|}{ Receipt of clinical follow-up: } \\
\hline Presented for clinical follow up & $9(6)$ & $6(22)$ \\
\hline \multicolumn{3}{|l|}{ Type of clinical follow up } \\
\hline Pap & $0(0)$ & $0(0)$ \\
\hline Colposcopy & $0(0)$ & $2(7)$ \\
\hline Cryotherapy & $9(6)$ & $4(15)$ \\
\hline Referral for surgery or palliation & $0(0)$ & $0(0)$ \\
\hline
\end{tabular}

HPV - human papillomavirus, VIA - visual inspection after acetic acid application
Among those who participated in hrHPV self-sampling, 56/159 (35\%) women had undergone previous screening. VIA was the most commonly reported modality $(37 / 56[66 \%])$, and most women reported that their prior results (51/56 [91\%]) were reported to them as normal. (Table 1 ).

\section{VIA and HPV Outcomes}

VIA, HPV, SMS text, and follow-up outcomes are listed in Table 2. Adequate VIA screening was achieved for 138 (87\%) women enrolled. Fourteen (10\%) were VIA positive, 114 (83\%) were VIA negative, and $8(6 \%)$ had an inconclusive or unsatisfactory result. Of the women who were VIA positive, nine (6\%) received cryotherapy treatment on site, and one (1\%) was referred to a higher level of care for a clinical impression of invasive cancer.

Of the 159 women who obtained HPV self-samples, 27 (17\%) were hrHPV positive, 120 (81\%) were hrHPV negative, and 4 (3\%) had an invalid result. Of the 27 women with hrHPV positive results, 6/27 (20\%) were positive for HPV 16, 6/27 (20\%) were positive for HPV 18 or 45 , and 21/27 (78\%) were positive for other high-risk HPV types. Co-infection with one or more high risk strain was present in five (19\%) women.

Among those with positive hrHPV results, 4 (17\%) also had positive findings on VIA, although none were suspicious for cancer. Three (11\%) underwent cryotherapy. HPV outcomes according to VIA results are included in Table 2.

\section{SMS Text Messages}

Nearly all (153 [96\%]) participants who obtained HPV self-samples provided a mobile phone number ( 15 of whom provided a smartphone). Most (126 [79\%]) participants were able to demonstrate literacy on enrollment, by completing enrollment surveys and reading alound SMS text messages options. SMS text messages were confirmed as delivered to 147 (93\%) participants (Figure 2). Six messages could not be delivered, none of which were intended for women with HPV positive results. Most women (139 [78\%]) were reachable by phone at the 6-month endpoint after SMS messages were sent. Of these, about half (74/139 [53\%]) reported receipt of messages, and 49 (50\%) denied receiving messages; 20 (13\%) could not be reached by phone (Table 2 ).

Of the 27 women with a hrHPV positive results, all of whom were sent SMS messages instructions to follow-up, 6 (22\%) followed up at the CCP for further screening, following receipt of text messages. Three women (11\%) received cryotherapy, 2 (4\%) had colposcopy and 1 (4\%) had a pap smear. All 27 women with hrHPV positive results were reached by phone at the six-month mark. The 21 women who had not yet presented for follow-up screening reported the following reasons: lack of transportation $(n=11)$, disbelief of results $(n=5)$, lack of childcare $(n=4)$, asymptomatic $(n=3)$, forgot $(n=3)$, avoidance of male provider and/or pelvic exam $(n=2)$, lack of trust in care providers $(n=1)$, perception of already receiving treatment at health fair $(\mathrm{n}=1)$.

\section{Acceptability}

Most women rated self-sampling highly and overall reported less embarrassment, less pain, higher confidence, and higher ease of screening with self-collected HPV compared with VIA screening (Table 3). The median differences, when compared to the standard deviation values, suggest large effect sizes, ranging from 0.6-0.7 standard deviation units for "confidence that screen performed correctly" to $>1$ standard deviation unit for "pain experienced during screening" and "likelihood to recommend screening" (Table 3). Individual Likert scale responses are included in Appendix S2 of the Online Supplementary Document. 


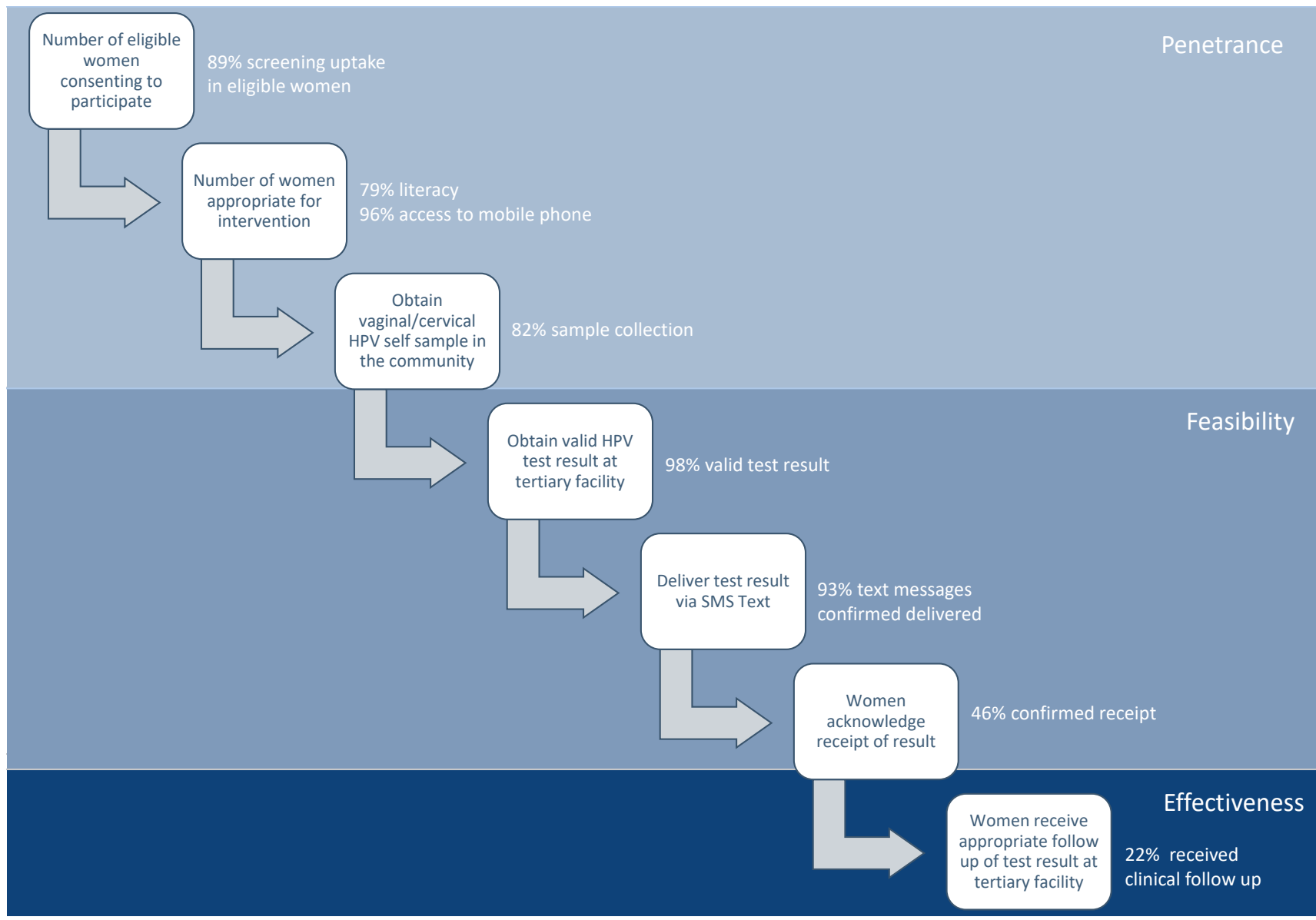

Figure 2. Nodes for effective linkage to care using community-based HPV self-sampling with tertiary hospital referral and outcomes. The flow diagram demonstrates key points for intervention and linkage for effectiveness in the cervical cancer screening cascade.

Table 3. Quality of self-collected HPV (median, SD) compared to visual inspection with acetic acid cervical cancer screening scale specific responses $(n=159)$

\begin{tabular}{|c|c|c|c|c|}
\hline & \multicolumn{4}{|c|}{ MEDIAN (SD) } \\
\hline & Self-collected & VIA & Effect size & $P$-value* \\
\hline Embarrassment felt during screening ( $1=$ very embarrassed, $5=$ not at all embarrassed) & $4.8(0.8)$ & $3.6(1.5)$ & $0.8-1.5$ & $<0.001$ \\
\hline Pain felt during screening ( 1 = severe discomfort, $5=$ no discomfort $)$ & $4.3(1.2)$ & $2.7(1.4)$ & $1.1-1.3$ & $<0.001$ \\
\hline Confidence that screen performed correctly ( $1=$ not at all confident, $5=$ very confident $)$ & $4.8(0.6)$ & $4.4(0.7)$ & $0.6-0.7$ & $<0.001$ \\
\hline Ease of performing screening during health fair ( $1=$ not that easy, $5=$ very easy $)$ & $4.6(0.6)$ & $3.8(1.1)$ & $0.9-1.5$ & $<0.001$ \\
\hline Likelihood to recommend screening ( 1 =very unlikely, 5 =highly likely) & $4.7(0.6)$ & $0.9(1.0)$ & $6.3-5.2$ & $<0.001$ \\
\hline
\end{tabular}

HPV - human papillomavirus, SD - standard deviation, VIA - visual inspection after acetic acid application

*Wilcoxon rank test used to compare responses.

\section{DISCUSSION}

In this study we piloted the implementation of community-based HPV self-sampling for primary cervical cancer screening in a rural agrarian-based population in Uganda, using SMS text messaging to notify study participants of their results. We achieved a high penetrance of eligible women attending the health fair (79\%). Most women were appropriate for the intervention, having demonstrated high rates of literacy (79\%) and access to a mobile phone (96\%). We demonstrated high feasibility in obtaining self-collected HPV samples, obtaining valid test results (98\%), and confirming delivery of SMS texts (93\%). However, result receipt by SMS was only confirmed as successful in about $50 \%$ of women who were reachable by phone at the 6-month endpoint, with only $22 \%$ of women with hrHPV positive results following up at the tertiary care facility (Figure 2). 
Our evaluation approach using the Proctor model enabled us to identify target areas in the cascade of cervical cancer screening for improvement of implementation. We demonstrated that self-sample collection can be performed with high penetrance, and that the context of the community is appropriate for an intervention based on SMS text result notification. However, there was high non-follow up rate in our participants. More work is needed to understand this finding, especially since SMS has been tested and demonstrated to successfully improve linkage to care among people living with HIV in the same catchment area as our study $[15,23]$. However in this study, this strategy for linkage to care was tested among people who had already been linked to HIV care at MRRH, participation in the study was restricted to those with confirmed cell phone ownership, and SMS messaging was combined with transportation reimbursement. In our study, participants may not have had engagement with, or previously visited, MRRH and were not provided funds for transportation to the clinic. Indeed, difficulty with transportation was cited as the most common reason for lack of follow-up. These findings demonstrate that SMS messaging may not fulfill the promise of improving linkage to care, and that other implementations gaps must be addressed in the cervical cancer screening cascade.

Primary prevention of cervical cancer hinges on successful vaccination. Currently available vaccines target HPV 16 and -18 genotypes [24]. In our cohort, although hrHPV prevalence was $21 \%$, HPV 16 prevalence was $2.7 \%$ and HPV $18 / 45$ prevalence was $1.9 \%$, which is similar to published findings from a large community based study in western Uganda [25]. This may have implications for the efficacy of vaccination in Uganda, and underscores the need for effective secondary prevention through screening.

Our findings should be interpreted in light of several limitations. Our cohort was limited to women who presented to the community health fair and who were willing to participate in cervical cancer screening,. Due to small sample size, we were unable to assess for predictors for follow-up. There was no control group to facilitate comparison in follow-up in those who did vs did not receive SMS follow-up and limited our ability to analyze the efficacy of text message follow-up. There was a 6-month interval from screening to result notification, which may have decreased the willingness of participants to engage in follow-up. This was done according to study protocol, given that the progression from infection to cancer occurs over decades, and given that all women who completed sampling had undergone a physical exam during VIA screening by experienced gynecologic oncology nurses and physicians; This was done according to study protocol, given that the progression from infection to cancer occurs over decades, and given that all women who completed sampling had undergone a physical exam during VIA screening by experienced gynecologic oncology nurses and physicians. It is also possible that women presented for follow-up care at health facilities other than MRRH, causing us to underestimate the rate of appropriate follow-up in this cohort. Finally, HPV self-sampling was performed concurrently with VIA screening at the time of the health fair. This was done to achieve a service delivery goal of cervical cancer screening using the current standard of care available. However, our simultaneous use of both methods of screening may have reduced willingness to follow-up among women receiving HPV positive results due to their perception of having already being screened.

Despite these limitations, our study presents a novel approach to community-based primary screening for cervical cancer and identifies key areas in the cervical cancer screening cascade and linkage to care that can be targeted for improvement. Though small in number, the willingness of women and reliance on community health workers for result delivery, unprompted by the study, reveals another potential avenue to achieve community-based screening, which may be promising. Further research will be need needed to examine the acceptability and performance of a community health worker-based HPV self-sampling strategy for community-based cervical cancer screening. This, coupled with strategies to reduce or cover transportation costs, either by providing travel reimbursements, or enabling follow-up screening at health facilities closer to women's homes, may improve success rates across the cascade of care.

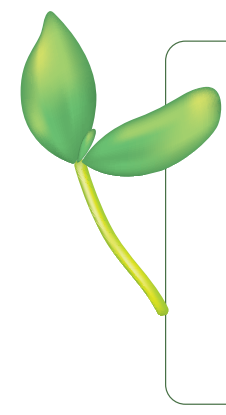

Acknowledgements: We thank the HopeNet cohort study participants, without whom this research would not be possible. We also thank members of the HopeNet study team for research assistance; in addition to the named study authors, HopeNet and other collaborative team members who contributed to data collection and/or study administration during all or any part of the study were as follows: Owen Alleluya, Dickson Beinomugisha, Bridget Burns, Patrick Gumisiriza, Clare Kamagara, Justus Kananura, Allen Kiconco, Viola Kyokunda, Patrick Lukwago Muleke, Rhina Mushagara, Rumbidzai Mushavi, Elijah Musinguzi, Moran Owembabazi, Elizabeth Betty Namara, Immaculate Ninsiima, Moran Owembabazi, Justin Rasmussen, Mellon Tayebwa, and Dagmar Vořechovská. We also thank Godfrey Massette, Phaisal Lubega, Flavia Ninsiima, and Patrick Orikiriza for additional research assistance. 
Funding: This work was made possible through funding from the Barry Foundation, Brigham \& Women's Hospital Connors Center for Women's Health and Gender Biology, the donation of HPV assay cartridges from Cepheid, Friends of a Healthy Uganda, and US National Institutes of Health R01MH113494. AAB is supported by career development awards from the Eunice Kennedy Schriever National Institute of Child Health and Human Development (K23HD097300) and Massachusetts General Hospital Executive Committee on Research through the Center for Diversity and Inclusion.

Author contributions: Initial conceptualization: NTJ, AN, PA, PA, JN, AAB. Refining study design: BK, CB, ACT, MJS, TRR. Data management: NTJ, AN, CB, MJ, AAB. Analysis: NTJ, ACT, TRR, JN, AAB. First draft: NTJ, TRR, AAB. Critical revision: $\mathrm{AN}, \mathrm{BK}, \mathrm{CB}, \mathrm{MJ}, \mathrm{PA}, \mathrm{PA}, \mathrm{ACT}, \mathrm{MJS}, \mathrm{JN}$.

Competing interests: ACT reports receiving a financial stipend from Elsevier, Inc. for his work as Co-editor in chief of the journal SSM-Mental Health. The authors have completed the ICMJE Declaration of Interest form (available upon request from the corresponding author) and declare no further conflicts of interest.

\section{Additional material}

Online Supplementary Document

1 Hull R, Mbele M, Makhafola T, Hicks C, Wang S-M, Reis RM, et al. Cervical cancer in low and middle-income countries. Oncol Lett. 2020;20:2058-74. Medline:32782524 doi:10.3892/ol.2020.11754

2 World Health Organisation. Global strategy to accelerate the elimination of cervical cancer as a public health problem and its associated goals and targets for the period 2020 - 2030. Vol. 2, United Nations General Assembly. 2020.

3 World Health Organization. Comprehensive cervical cancer control: A guide to essential practice. Geneva: WHO; 2014.

4 Gakidou E, Nordhagen S, Obermeyer Z. Coverage of cervical cancer screening in 57 countries: Low average levels and large inequalities. PLoS Med. 2008;5:e132. Medline:18563963 doi:10.1371/journal.pmed.0050132

5 Lemp JM, De Neve J-W, Bussmann H, Chen S, Manne-Goehler J, Theilmann M, et al. Lifetime Prevalence of Cervical Cancer Screening in 55 Low- and Middle-Income Countries. JAMA. 2020;324:1532-42. Medline:33079153 doi:10.1001/ jama.2020.16244

6 Pierz AJ, Randall TC, Castle PE, Adedimeji A, Ingabire C, Kubwimana G, et al. A scoping review: Facilitators and barriers of cervical cancer screening and early diagnosis of breast cancer in Sub-Saharan African health settings. Gynecol Oncol Rep. 2020;33:100605. Medline:32637528 doi:10.1016/j.gore.2020.100605

7 Ronco G, Giorgi-Rossi P, Carozzi F, Confortini M, Palma PD, Del Mistro A, et al. Efficacy of human papillomavirus testing for the detection of invasive cervical cancers and cervical intraepithelial neoplasia: a randomised controlled trial. Lancet Oncol. 2010;11:249-57. Medline:20089449 doi:10.1016/S1470-2045(09)70360-2

8 Rijkaart DC, Berkhof J, Rozendaal L, van Kemenade FJ, Bulkmans NWJ, Heideman DAM, et al. Human papillomavirus testing for the detection of high-grade cervical intraepithelial neoplasia and cancer: final results of the POBASCAM randomised controlled trial. Lancet Oncol. 2012;13:78-88. Medline:22177579 doi:10.1016/S1470-2045(11)70296-0

9 Campos NG, Castle PE, Wright TC Jr, Kim JJ. Cervical cancer screening in low-resource settings: A cost-effectiveness framework for valuing tradeoffs between test performance and program coverage. Int J Cancer. 2015;137:2208-19. Medline:25943074 doi:10.1002/ijc.29594

10 Catarino R, Vassilakos P, Bilancioni A, Bougel S, Boukrid M, Meyer-Hamme U, et al. Accuracy of self-collected vaginal dry swabs using the Xpert human papillomavirus assay. PLoS One. 2017;12:e0181905. Medline:28750015 doi:10.1371/journal. pone.0181905

11 Arbyn M, Verdoodt F, Snijders PJF, Verhoef VMJ, Suonio E, Dillner L, et al. Accuracy of human papillomavirus testing on self-collected versus clinician-collected samples: a meta-analysis. Lancet Oncol. 2014;15:172-83. Medline:24433684 doi:10.1016/ S1470-2045(13)70570-9

12 Polman NJ, Ebisch RMF, Heideman DAM, Melchers WJG, Bekkers RLM, Molijn AC, et al. Performance of human papillomavirus testing on self-collected versus clinician-collected samples for the detection of cervical intraepithelial neoplasia of grade 2 or worse: a randomised, paired screen-positive, non-inferiority trial. Lancet Oncol. 2019;20:229-38. Medline:30658933 doi:10.1016/S1470-2045(18)30763-0

13 World Health Organization. Planning and implementing cervical cancer prevention and control programs: Manual for managers. Geneva: WHO. 2004.

14 Nakalembe M, Makanga P, Kambugu A, Laker-Oketta M, Huchko MJ, Martin J. A public health approach to cervical cancer screening in Africa through community-based self-administered HPV testing and mobile treatment provision. Cancer Med. 2020;9:8701-12. Medline:32966684 doi:10.1002/cam4.3468

15 Siedner MJ, Lankowski A, Musinga D, Jackson J, Muzoora C, Hunt PW, et al. Optimizing Network Connectivity for Mobile Health Technologies in sub-Saharan Africa. PLoS One. 2012;7:e45643. Medline:23029155 doi:10.1371/journal.pone.0045643

16 Horvath T, Azman H, Kennedy GE, Rutherford GW. Mobile phone text messaging for promoting adherence to antiretroviral therapy in patients with HIV infection. Cochrane Database Syst Rev. 2012;2012:CD009756. Medline:22419345 doi:10.1002/14651858.CD009756

17 Palmer MJ, Henschke N, Villanueva G, Maayan N, Bergman H, Glenton C, et al. Targeted client communication via mobile devices for improving sexual and reproductive health. Cochrane Database Syst Rev. 2020;CD013680. Medline:32779730 
18 Kakuhikire B, Satinsky EN, Baguma C, Rasmussen JD, Perkins JM, Gumisiriza P, et al. Correlates of attendance at community engagement meetings held in advance of bio-behavioral research studies: A longitudinal, sociocentric social network study in rural Uganda. PLoS Med. 2021;18:e1003705. Medline:34270581 doi:10.1371/journal.pmed.1003705

19 World Health Organization. WHO Guidelines for Screening and Treatment of Precancerous Lesions for Cervical Cancer Prevention. Geneva: WHO; 2013.

20 Nakisige C, Schwartz M, Ndira AO. Cervical cancer screening and treatment in Uganda. Gynecol Oncol Rep. 2017;20:37-40. Medline:28275695 doi:10.1016/j.gore.2017.01.009

21 Proctor EK, Landsverk J, Aarons G, Chambers D, Glisson C, Mittman B. Implementation research in mental health services: an emerging science with conceptual, methodological, and training challenges. Adm Policy Ment Health. 2009;36:24-34. Medline:19104929 doi:10.1007/s10488-008-0197-4

22 Harris PA, Taylor R, Thielke R, Payne J, Gonzalez N, Conde JG. Research electronic data capture (REDCap)-a metadata-driven methodology and workflow process for providing translational research informatics support. J Biomed Inform. 2009;42:37781. Medline:18929686 doi:10.1016/j.jbi.2008.08.010

23 Siedner MJ, Santorino D, Lankowski AJ, Kanyesigye M, Bwana MB, Haberer JE, et al. A combination SMS and transportation reimbursement intervention to improve HIV care following abnormal CD4 test results in rural Uganda: a prospective observational cohort study. BMC Med. 2015;13:160. Medline:26149722 doi:10.1186/s12916-015-0397-1

24 van Bogaert L. Are the currently existing anti-human papillomavirus vaccines appropriate for the developing world? Ann Med Health Sci Res. 2013;3:306-12. Medline:24116304 doi:10.4103/2141-9248.117924

25 Nakalembe M, Makanga P, Mubiru F, Swanson M, Martin J, Huchko M. Prevalence, correlates, and predictive value of high-risk human papillomavirus mRNA detection in a community-based cervical cancer screening program in western Uganda. Infect Agent Cancer. 2019;14:14. Medline:31114629 doi:10.1186/s13027-019-0230-0 